\title{
INTERVAL CLANS WITH NONDEGENERATE KERNEL ${ }^{1}$
}

\author{
R. C. PHILLIPS
}

Introduction. The object of this paper is to characterize the clans (compact connected Hausdorff topological semigroups with an identity element) which are homeomorphic to a unit interval and which have a nondegenerate kernel (minimal two-sided ideal). The corresponding case when the kernel is degenerate has been characterized in a paper by $\mathrm{H}$. Cohen and L. I. Wade [2] together with an earlier paper by Mostert and Shields [5].

In a topological semigroup $T, K(T)$ or $K$ denotes the kernel of $T$. The symbol $u$ is reserved to denote an identity element. The term "standard thread" will mean a clan with zero which is homeomorphic to a unit interval and whose endpoints are its zero and identity element. In a standard thread $T$ with identity element $u$ and zero 0 , for $a, b \in T,[a, b]$ will denote the interval from $a$ to $b$, (or $b$ to $a$ ) inclusive and $a \leqq b$ will mean $a \in[0, b]$, with $a<b$ in case $a \neq b$. A relation $R$ on a topological semigroup $x$ is called a "closed right congruence" if (i) $R$ is an equivalence relation, (ii) $a, b, c \in X, a R b$ implies $a c R b c$, (iii) $a R x_{n}$ for $n=1,2, \cdots$ and $x_{n} \rightarrow x$ implies $a R x$ (closed). We denote by $R_{a}$ the set $\{x \mid a R x\}$. The analog of Theorem I where $R$ satisfies $(2)^{\prime}(a, b, c \in X, a R b$ implies $c a R c b$ (left congruence) instead of (2) is also true.

I would like to express my appreciation to Professor H. Cohen and Professor R. J. Koch for their assistance in the preparation of this paper.

TheOREM I. Let $T$ be a standard thread and $R_{a}$ closed right congruence on $T$. Then for $a \in T$ either (1) $R_{a}=a$ or (2) $R_{a}$ is an interval $[e, b]$ where $e$ is idempotent and $[e, b]$ is a subsemigroup of $T$ with zero element $e$.

Proof. Suppose there exists $a^{\prime} \in T$ such that $a^{\prime} R a$ and $a^{\prime} \neq a$. Let $e=\inf \{x: x R a\}$ and $b=\sup \{x: x R a\}$. Since $R_{a}$ is closed, $e R a$ and $b R a$. Now $e<b$ which implies [3] that $e=b r$ for some $r \geqq e$. Therefore, $b$ Re implies brRer implies $e$ Rer implies $e \operatorname{Rer}^{n}$ for $n=1,2, \cdots$. From [3] we know $r^{n} \rightarrow j=j^{2} \leqq r$ and hence $e R e j$. We will show $j \leqq e$. If $j \geqq b$, then $b j=b$ and hence $b=b j=b(j r)=(b j) r=b r=e$. Therefore $j<b$

Received by the editors, April 2, 1962.

1 This research was supported by the United States Air Force through the Air Force Office of Scientific Research and Development Command, under Contract AF 189(603-89). 
and $j=b j \leqq b r=e$, as was to be shown. Now $j \leqq e$ implies $e j=j$ and since $e R e j, e R j$ which implies by the minimality of $e$, that $e=j$ and hence $e^{2}=e$. Now for $c \in[e, b], c=b p$ for some $p \geqq c$ and since $e R b$, $e p R b p$ and we have $e R c$. This shows that $R_{a}=[e, b]$ which is indeed a semigroup with zero element $e$.

Notice that if $X$ and $Y$ are topological semigroups and $f$ maps $X$ into $Y$ continuously and either $f(x y)=f(x) f(y)$ or $f(x y)=f(x) \cdot y$, (in case $Y$ is contained in $X$ ), then the relation $R$ induced by letting $R_{a}=f^{-1} f(a)$ is a closed right congruence on $X$.

Corollary II. Let $X$ be a topological semigroup and let $A$ be a standard thread contained in $X$. Then for any element $c \in X, c A$ is a continuous monotone image of $A$.

Proof. Define the relation $R$ on $A$ by $R_{a}=\{x \mid x \in A, c x=c a\}$.Then $R$ is a closed right congruence and hence multiplication by $c$ is monotone.

Clans on an interval. Let $S$ be a clan which is homeomorphic to a unit interval and which has a nondegenerate kernel, $K$. By a result of A. D. Wallace [7], the identity element $u$ of $S$ is one of the endpoints of $S$. Note that $K$ is a closed interval of $S$ and let (i) $A=$ the closure of the component of $S-K$ which contains $u$, (ii) $B=S$ $-\left(K^{0} \cup A\right)$, (iii) $z=A \cap K$, (iv) $z^{\prime}=B \cap K$, and (v) $d=$ the nonidentity endpoint of $S$. A result of Faucett [4] is that $A$ is an abelian subclan of $S$ with zero $z$ and that $K$ consists of either all left zeroes or all right zeroes of $S$. Let us assume that $K$ is all right zeroes.

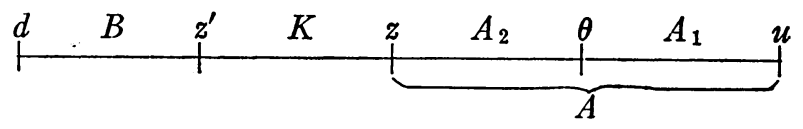

FIGURE I

LEMMA III. $A B=A d=B$.

Proof. Since $A$ is a standard thread contained in $S$, by the analog of Corollary II, $A d$ is a continuous monotone image of $A$ and $A d$ $=[z d, u d]=[z d, d]$ which contains $B$ since $z d$ is in $K$. In particular, there exists an element $a$ in $A$ such that $z^{\prime}=a d$. Now since $z$ is the zero for $A, z=z a$ and we have $z d=(z a) d=z(a d)=z z^{\prime}=z^{\prime}$. Therefore $A d=\left[d, z^{\prime}\right]=B$. Also $A B=A(A d)=A^{2} d=A d=B$ as was to be shown.

Notice that from Corollary II we have $d A=[d z, d u]=[z, d]$ which contains $z^{\prime}$. Let $\theta=\inf \left\{a: a \in A, d a=z^{\prime}\right\}$. Denote $[\theta, u]$ by $A_{1}$ and $[z, \theta]$ by $A_{2}$. (Note that $\theta \neq z$, else $z^{\prime}=d \theta=z$.)

LEMmA IV. $B A_{1}=d A_{1}=B ; B A_{2}=d A_{2}=K$; and $\theta^{2}=\theta$. 
Proof. In the same manner as the proof of Lemma III we have $d A_{1}=\left[z^{\prime}, d\right]=B$ and $d A_{2}=\left[z^{\prime}, z\right]=K$. Since $A_{2}^{2} \subset A_{2}[3], \theta^{2} \in A_{2}$. Therefore $d \theta^{2} \in d A_{2}=K$. But $d \theta^{2}=z^{\prime} \theta \in B A_{1}=A d A_{1}=A B=B$. So $d \theta^{2} \in K \cap B=z^{\prime}$ and by the minimality of $\theta, \theta^{2}=\theta$. Now we employ a result of Mostert and Shields [5] that $A_{1}$ and $A_{2}$ are subclans of $A$, that $\theta$ is a zero for $A_{1}$ and an identity for $A_{2}$, and that $a \in A_{1}, a^{\prime} \in A_{2}$ implies $a a^{\prime}=a^{\prime} a=a^{\prime}$. Therefore $A_{1} A_{2}=A_{2}$ and $B A_{2}=d A_{1} A_{2}=K$ and the lemma is proved.

LemмA V. $B$ is an abelian subsemigroup of $S$.

Proof. First we show $d^{2}$ is in $B$. Clearly $d^{2}$ is not in $A$, else $z^{\prime}=d z^{\prime}$ $=d(d \theta)=d^{2} \theta \in A$. So suppose $d^{2}$ is in $K$. Then $d d^{2}=d^{2}$ and by Lemma IV $d^{2}=d a$ for some $a$ in $A_{2}$. Therefore $d^{2} u=d^{2}=d d^{2}=d(d a)=d^{2} a$, and since left multiplication by $d^{2}$ induces a closed right congruence on $[z, u], d^{2}[u, a]=d^{2}$. Now $a \in A_{2}$ so that $\theta$ is in $[u, a]$. Hence $d^{2} \theta=d^{2}$. But $d^{2} \theta=d(d \theta)=d z^{\prime}=z^{\prime}$ and $d^{2}$ is in $B$. Using Lemmas III and IV, we have $B^{2}=\left(d A_{1}\right)\left(d A_{1}\right) \subset d B A_{1}=d\left(d A_{1}\right) \subset B A_{1}=B$, i.e., $B^{2} \subset B$. Now we show $B$ is abelian. Using again Lemmas III and IV, $a \in A_{1}$ implies $d a \in B$ and $d a=a^{\prime} d$ for some $a^{\prime} \in A$. Since $d^{2} \in B, d^{2}=a^{\prime \prime} d$ for some $a^{\prime \prime} \in A$. Using the commutativity of $A$, we have $d a d=\left(a^{\prime} d\right) d=a^{\prime} a^{\prime \prime} d$ $=a^{\prime \prime} a^{\prime} d=a^{\prime \prime} d a=d^{2} a$. Let $b, b^{\prime}$ be elements of $B$. Then for some $a_{1}, a_{2} \in A_{1}, b=d a_{1}$, and $b^{\prime}=d a_{2}$. So $b b^{\prime}=d a_{1} d a_{2}=d^{2} a_{1} a_{2}=d^{2} a_{2} a_{1}=d a_{2} d a_{1}$ $=b^{\prime} b$ and $B$ is abelian.

LEMMA VI. $K B=\boldsymbol{z}^{\prime}$.

Proof. Since $K B=d A_{2} d A_{1}=d\left(A_{2} d\right) A_{1} \subset d B A_{1}=d B \subset B, K B \subset K$ $\cap B=z^{\prime}$.

In what follows $S / K$ denotes the Rees quotient [6] of $S$ modulo $K$ and $F$ denotes the natural map of $S$ onto $S / K$. Since $d^{2} \in B, F\left(d^{2}\right)$ $\in F(B)$ and we have

THEOREM VII. Let $S$ be an interval clan with a nondegenerate kernel $K$. Let $u$ be the identity and $d$ the nonidentity endpoint of $S / K$; denote $F(K)$ by 0 . Then (i) there exists an element $\theta=\theta^{2} \in[0, u]-\{0\}$ such that $d \theta=0$ and (ii) $d^{2} \in[d, 0]$. Further, the function $h: F\left(A_{2}\right) \rightarrow K$ defined by $h(x)=d \cdot F^{-1}(x)$ for $x \neq 0$ and $h(0)=z$ is continuous and induces a closed right congruence on $F\left(A_{2}\right)$.

Let $S$ be a clan on an interval $[d, u]$ where $u$ is the identity element. Suppose $S$ has a zero 0 , that (i) and (ii) of Theorem VII are satisfied, that $d \neq 0$ and that $u \neq \theta$. Consider the real interval $[1,5]$ and define

1. $f:[d, 0] \rightarrow[1,2]$ so that $f(d)=1, f(0)=2$ and $f$ is a homeomorphism, 
2. $g:[0, u] \rightarrow[3,5]$ so that $g(0)=3, g(\theta)=4, g(u)=5$ and $g$ is a homeomorphism,

3. $h:[0, \theta] \rightarrow[2,3]$ so that $h(0)=3, h(\theta)=2, h$ is continuous and $h$ induces a closed right congruence $R$ on $[0, \theta],\left(R_{x}=h^{-1} h(x)\right)$.

Further, define for all $x, y$ and $z$ on which the functions are defined,

4. $c \cdot h(x)=h(x)$, all $c \in[1,5]$,

5. $h(x) \cdot g(y)=h(x y)$,

6. $h(x) \cdot f(y)=2$,

7. $g(x) \cdot g(y)=g(x y)$,

8. $f(x) \cdot f(y)=f(x y)$,

9. $g(x) \cdot f(y)=f(x y)$,

10. $f(x) \cdot g(y)=\{h(y)$ for $y \in[0, \theta], f(x y)$ for $y \in[\theta, u]\}$.

We now show that definition 5 is well defined. The others are clear. Suppose $h(a)=h(b)$ and $a<b$. Then from condition 3 and Theorem I we have $a, b \in[e, r]=h^{-1} h(x)$ and $e^{2}=e$ and $e$ is a zero for $[e, r]$, a semigroup. Then for $c \in[0, u], h(a) \cdot g(c)=h(a c)$ and $h(b) \cdot g(c)=h(b c)$. If $c \leqq e$, then $a c=b c=c[5]$ so that $h(a c)=h(b c)$. If $c>e$, then $e \leqq a c \leqq a$ and $e \leqq b c \leqq b$ and $h(a c)=h(b c)=h(a)$, and $h$ is well defined.

It can be easily verified that the interval $[1,5]$ together with definitions 4 through 10 is a clan $S^{\prime}$ with kernel $[2,3]$ and that $S^{\prime} / K$ is topologically isomorphic to $S$.

Now suppose $S$ in the previous construction were the Rees quotient of an interval clan $T=\left[d, z^{\prime}, z, u\right]$ with nondegenerate kernel $K=\left[z^{\prime}, z\right]$. Then outside $K, T$ is reproduced by our construction and if $h$ in 3 is chosen to be the $h$ of Theorem VII, the resulting clan is topologically isomorphic to $T$.

If $d=0$, omit definitions $1,6,8,9$, and 10 ; if also $u=\theta$, omit the equation $g(u)=5$ from definition 2 ; change definition 4 appropriately. The conclusion is completely analogous for $S=[2,4]$ if $u=\theta$ or $S=[2,5]$ if $u \neq \theta$.

From the preceding two paragraphs we conclude:

TheOREM VIII. An interval clan with a nondegenerate kernel is characterized by a pair $(S, h)$ where

1. $S$ is an interval clan with zero 0 , say $S=[d, 0, u]$ where $d$ may equal 0 ,

2. $d^{2} \in[d, 0]$,

3. there exists $\theta=\theta^{2} \in(0, u]$ such that $d \theta=0$,

4. $h$ maps $[0, \theta]$ onto $[1,2]$ continuously,

5. $h$ induces a closed right (left in case $K$ is all left zeroes) congruence on $[0, \theta]$.

The pair $(S, h)$ characterizes a particular interval clan $T$ with a 
nondegenerate kernel $K$ in the following sense; $T / K=S$ and the function $h$ of Theorem VII satisfy the conditions of Theorem VIII, and a clan $T^{\prime}$ gives rise to the same $S$ and $h$ if and only if $T^{\prime}$ is topologically isomorphic to $T$.

\section{BIBLIOGRAPHY}

1. A. H. Clifford, Semigroups containing minimal ideals, Amer. J. Math. 70 (1948), 521-526.

2. Haskell Cohen and L. I. Wade, Clans with zero on an interval, Trans. Amer. Math. Soc. 88 (1958), 523-535.

3. W. M. Faucett, Compact semigroups irreducibly connected between two idempotents, Proc. Amer. Math. Soc. 6 (1955), 741-747.

4. - Topological semigroups and continua with cutpoints, Proc. Amer. Math. Soc. 6 (1955), 708-756.

5. P. S. Mostert and A. L. Shields, On the structure of semigroups on a compact manifold with boundary, Ann. of Math. (2) 65 (1957), 117-143.

6. D. Rees, On semigroups, Proc. Cambridge Philos. Soc. 36 (1940), 387-400.

7. A. D. Wallace, The structure of topological semigroups, Bull. Amer. Math. Soc. 61 (1955), 95-112.

Louisiana State University 